\title{
Fluctuating Elasticity Mode in Transient Molecular Networks
}

\author{
Giovanni Nava, ${ }^{1}$ Marina Rossi, ${ }^{1}$ Silvia Biffi, ${ }^{1}$ Francesco Sciortino, ${ }^{2}$ and Tommaso Bellini ${ }^{1, *}$ \\ ${ }^{1}$ Department of Medical Biotechnology and Translational Medicine, Università degli Studi di Milano, \\ via Fratelli Cervi 93, I-20090 Segrate, Milano, Italy \\ ${ }^{2}$ Department of Physics and CNR-ISC, Sapienza Università di Roma, Piazzale Aldo Moro 2, I-00185 Roma, Italy
}

(Received 19 April 2017; published 16 August 2017)

\begin{abstract}
Transient molecular networks, a class of adaptive soft materials with remarkable application potential, display complex, and intriguing dynamic behavior. By performing dynamic light scattering on a wide angular range, we study the relaxation dynamics of a reversible network formed by DNA tetravalent nanoparticles, finding a slow relaxation mode that is wave vector independent at large $q$ and crosses over to a standard $q^{-2}$ viscoelastic relaxation at low $q$. Exploiting the controlled properties of our DNA network, we attribute this mode to fluctuations in local elasticity induced by connectivity rearrangement. We propose a simple beads and springs model that captures the basic features of this $q^{0}$ behavior.
\end{abstract}

DOI: 10.1103/PhysRevLett.119.078002

Introduction.-Transient molecular networks are systems in which colloidal particles, molecular aggregates, or polymers reversibly bind into percolating tridimensional matrices capable of sustaining stress. Reversible bonds can be provided by soft molecular interactions (hydrophobic, electrostatic, $\mathrm{H}$ bonds) or by conformational constraints, as in the case of entangled polymer solutions [1]. Despite the diversity of the systems in this class, transient molecular networks share basic mechanical properties that make them relevant as adaptive, reversible, self-healing materials, applications for which they are currently under intense scrutiny $[2,3]$.

Transient molecular networks also share complex dynamic behavior, generally dominated by two main distinct and coexisting processes, as observed either in the relaxation after mechanical, electrical, or optical stimuli [4-6] or by studying spontaneous fluctuations. The study of concentration fluctuation has enabled determining, in some of these systems, the characteristic time of the two processes as a function of the wave vector $q$ [7-15]. The faster mode $\tau_{F}$ is generally found to scale as $\tau_{F} \propto q^{-2}$, as typical of collective diffusion. During this process, concentration inhomogeneities relax only partially. Full decorrelation is achieved only after a longer time $\tau_{S}$. Intriguingly, such slower relaxation displays different features in different systems and in different regimes of the same system. Some studies on microemulsions [7], semidilute solutions of polymer [8-10], or block copolymers [11] report that the slower process scales as $\tau_{S} \propto q^{-2}$, which is attributed to topological rearrangement, viscoelastic decay or cluster formation, respectively. Another group of experiments in rodlike micelles [12], semidilute polymers with bonding agents [8,9,13], telechelic ionomers [14] reported instead a $q$-independent slow mode. In a few cases the slower mode appears to change from $q^{-2}$ to " $q$ " the polymer concentration $[8,9,15]$. What the microscopic mechanism behind such a $q^{0}$ mode is and which phenomena control the range of conditions where it is found, still need a convincing explanation.

Transient networks are typically composed by rather complex units which allow neither a precise knowledge of the node structure and size nor a control of the internode bond lifetime. Those elements appear instead to be the ingredients essential to unveil the molecular processes implied in such unconventional dynamics. We present here a study of the collective dynamics of a DNA-based transient network of which we design particles, connectivity, bond strength, and bond lifetime. The network connectivity is produced by the reversible binding of DNA multistrand constructs shaped as four-arms nanostars (NS) with sticky tips, a realization of low-valence colloids [16,17] (see the Supplemental Material [18]). When sufficiently concentrated, NS dispersions undergo a continuous reversible transformation from a fluid of independent particles to a gel in a small temperature $(T)$ range by passing through a continuum of equilibrium states [19]. Thus, this system enables exploring the dynamics of a transient network in a huge range of network strengths and bond lifetimes, which is something that cannot be easily done in the other transient molecular networks. By performing light scattering in a wide range of wave vectors that extends those of traditional approaches to values obtained by small angle detection, we could observe the crossover of the $q^{0}$ mode to $q^{-2}$ and its $T$ dependence. The specificity of this system enables us to clearly differentiate the role of the network in the dynamical processes and understand the slower mode as a product of the topological rearrangements bringing about fluctuation in the local bulk modulus. Accordingly, we propose here a zeroth order model based on elasticity fluctuations, which enables us to explain all the basic features of the $q^{0}$ mode.

Methods.-A solution of DNA NS at concentration $c=21.3 \mathrm{mg} / \mathrm{ml}(355 \mu \mathrm{M})$ and ionic strength of $50 \mathrm{mM}$ $\mathrm{NaCl}$ was investigated by means of dynamic light 


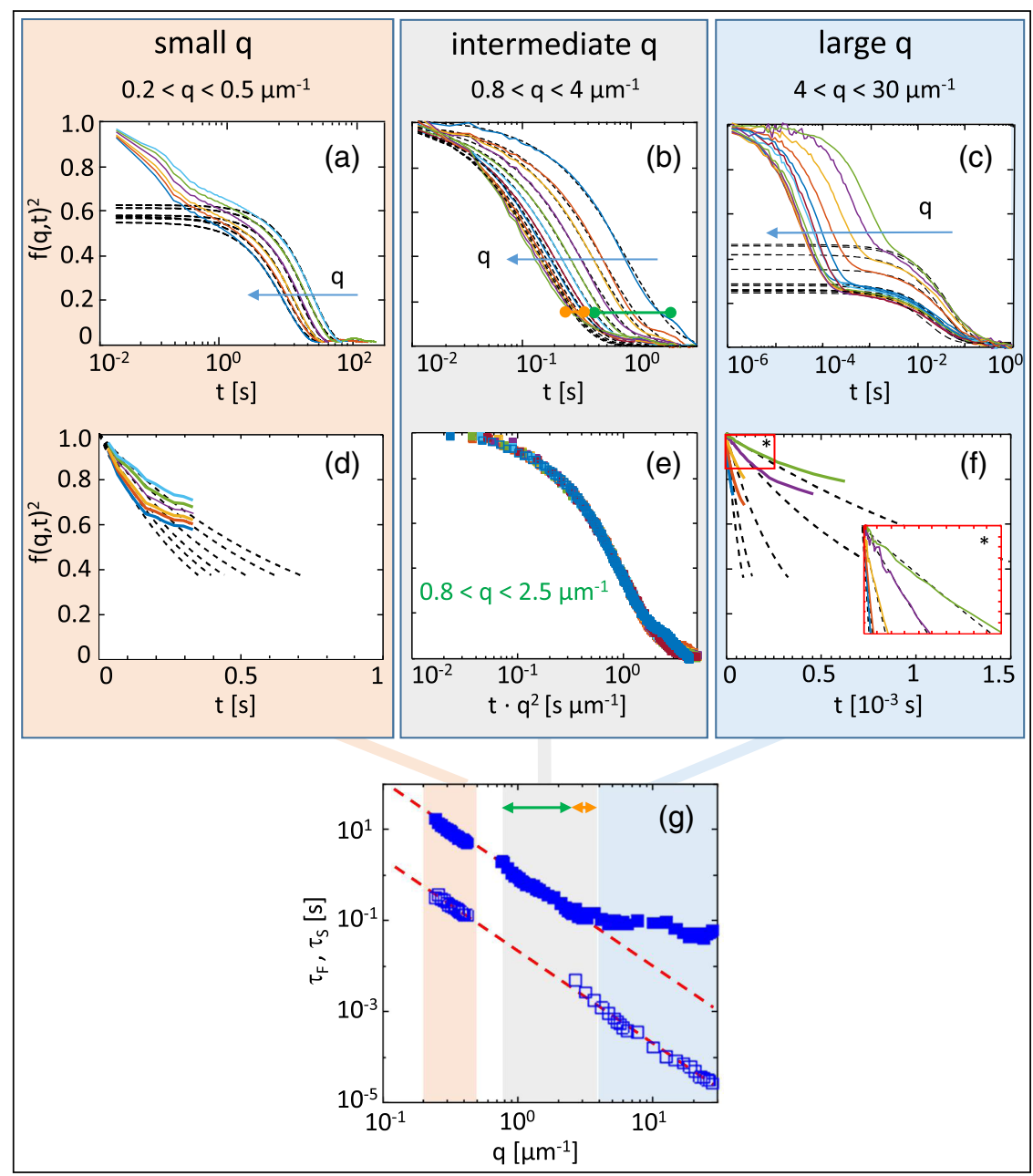

FIG. 1. Squared intermediate scattering function $f(q, t)^{2}$ measured at $T=27^{\circ} \mathrm{C}$ (continuous colored lines) in the small $q$ (pink shading), intermediate $q$ (gray shading), and large $q$ (blue shading) intervals, normalized either to $\tau=0$ (a),(c) or to the amplitude of the slow decay (b). Distinct colors indicate different $q$, with $q$ increasing as indicated by the arrows. Dashed lines: stretched exponential fits to the slower component of the correlations [(a)-(c)]; exponential fits to the faster component of the decay in the small $q$ (d) and large $q$ [(f) and inset] ranges. Orange and green marks in pane (b) indicate two subsets of correlations, the latter plotted in pane (e) vs $\tau q^{2}$. Characteristic times for the fast (full symbols) and slow modes (open symbols) and $q^{-2}$ lines are plot in pane $(\mathrm{g})$.

scattering (DLS) in the range $30 \mu \mathrm{m}^{-1}<q<0.2 \mu \mathrm{m}^{-1}$, corresponding to length scales ranging from hundreds to tens of thousands times the mean NS distance. In this interval we determined the intermediate scattering function $f(q, t)$ defined as

$g_{2}(q, t)=\left\langle I\left(q, t^{\prime}\right) I\left(q, t^{\prime}+t\right)\right\rangle_{t^{\prime}} /\langle I(q)\rangle_{t^{\prime}}^{2}=1+b[f(q, t)]^{2}$,

where $g_{2}(q, t)$ is the time correlation function for the scattered intensity $I(q, t)$ at a scattering vector $q$ and lag time $t . b$ is the Siegert coefficient, which depends on the experimental setup. Averages are performed over time.

To access a $q$ range functional to a clear experimental description of the dynamics of this system, we realized a custom setup that combines three distinct detection schemes corresponding to three different wave vector $q$ ranges: Large $q$ " $L q$ " $\left(4 \mu \mathrm{m}^{-1}>q>30 \mu \mathrm{m}^{-1}\right)$, Intermediate $q$ " $I q$ " $\left(0.8 \mu \mathrm{m}^{-1}<q<4 \mu \mathrm{m}^{-1}\right)$, and small $q$ " $S q$ " $\left(0.2 \mu \mathrm{m}^{-1}<\right.$ $q<0.5 \mu \mathrm{m}^{-1}$ ) (see the Supplemental Material [18]).

Measurements were performed after at least $3 \mathrm{~h}$ of thermalization (see the Supplemental Material [18]) at three temperatures: $T=35^{\circ} \mathrm{C}, T=27^{\circ} \mathrm{C}$, and $T=15^{\circ} \mathrm{C}$.
Results and discussion.-Figures 1(a)-1(c) show $f(q, t)^{2}$ measured at $T=27^{\circ} \mathrm{C}$ in the $S q, I q$, and $L q$ ranges, respectively. A double decay is clearly detectable in the $S q$ and $L q$ ranges. In the $I q$ data only the slower process is visible because of the technical limitation on the shortest lag times (see the Supplemental Material [18]). The correlations in the figures are normalized to their amplitude at $t=0$ (in $L q$ and $S q$ ) or to the amplitude of the slow mode only (in $I q$ ).

Correlation curves in Figs. 1(a)-1(c) can be analyzed to determine the $q$ dependence of the characteristic time of both the fast $\left(\tau_{F}\right)$ and slow $\left(\tau_{S}\right)$ components (see the Supplemental Material [18]). The slow relaxation can be well fitted by a stretched exponential function $f(q, t)=$ $h(q) \exp \left[-\left(t / \tau_{s}\right)^{0.8}\right]$ as it can be appreciated in the figures where the best fitting curves are plotted as dashed lines. The value of $\tau_{S}$ at $27^{\circ} \mathrm{C}$ thus determined are plotted in Fig. 1(g) (full symbols) where the shading marks the three regimes. At large enough $q, \tau_{S}$ is nearly $q$ independent for about a decade in $q$, limited by the largest accessible $q$. As $q$ decreases below $3 \mu \mathrm{m}^{-1}$, $\tau_{S}$ becomes $q$ dependent and adopts a $\tau_{S}=A_{S} q^{-2}$ scaling, with coefficient $A_{S} \approx$; $10^{12} \mathrm{~s} / \mathrm{m}^{2} . h(q)$, the amplitude of the slow mode relative to the whole relaxation, grows from $h \approx 0.5$ to $h \approx 0.9$ upon lowering $q$ (see Fig. 2 in the Supplemental Material [18]). 


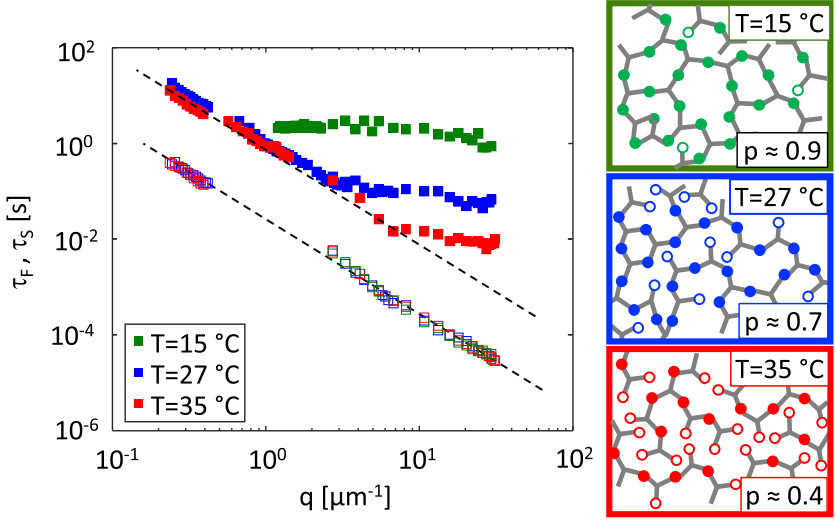

FIG. 2. Relaxation times of the fast mode ( $\tau_{F}$, empty symbols) and of the slow mode ( $\tau_{S}$, full symbols) at various $\mathrm{T}$ as a function of $q$. Sketches on the right-hand side (where DNA NS are pictured as gray three-arms stars) are aimed to mimic the gel's aggregation state at the temperatures here considered, where $p$ is the fraction of bound terminals (solid dots: bound terminals, open dots: unbound terminals). At the three $T$, the bond lifetimes are also different, ranging from short-lived (red dots) to long-lived (green dots).

An analogous crossover from $q^{0}$ at larger $q$, to $q^{-2}$ at smaller $q$, is observed for the two other $T$ considered in this study. The values of $\tau_{S}(q, T)$ for the three temperatures are plotted in Fig. 2 (full symbols). In the " $q^{0}$ regime" where $\tau_{S}$ appears to be $q$ independent, $\tau_{S}$ can be approximated by a power law dependence $\tau_{S} \sim q^{-\alpha}$ with $0<\alpha<0.3$. In this regime, $\tau_{S}$ grows with decreasing $T$ as an Arrhenius law, $\tau_{S}=\tau_{S 0} \exp \left(\Delta H / k_{B} T\right)$ with an activation energy $\Delta \mathrm{H} \approx ; 120 \pm 5 \mathrm{kcal} / \mathrm{mol}$ approximately matching the enthalpy required to open three bonds between NSs [19] (see the Supplemental Material [18]). $\tau_{S}$ is therefore proportional to the lifetime of inter-NS bond. At the opposite limit of small $q$, we find that the coefficient $A_{S}$ has a very weak dependence on $T$, if any. The wave vector at which the crossover to the $q^{-2}$ occurs is thus strongly $T$ dependent, as visible in Fig. 2, with $q_{c} \approx \sqrt{\tau_{S 0} / A_{S}} \exp \left(\Delta H / 2 k_{B} T\right)$. The crossover can be directly appreciated in the $I q$ range at $T=27^{\circ} \mathrm{C}$ in Fig. 1(b) where the subset of $f(q, t)^{2}$ in the upper range of the $I q$ interval (orange mark) overlap, indicating equal $\tau_{S}$, while at lower $q$ (green mark) they are spread, indicating a $q$-dependent $\tau_{S}$. When plotted vs $t q^{2}$ only this last group of correlations collapses [Fig. 1(e)].

Since $\tau_{S}$ in the $q^{0}$ range marks the inter-NS bond lifetimes, the kinetic behavior observed at shorter times must not involve the disruption of bonds but rather reflect concentration fluctuations occurring at fixed network topology. Such a process is reminiscent of the caging effect in concentrated colloids, although in this case the diffusional freedom is not set by the size of the cage but by the flexibility of the network. The characteristic time of these fluctuations at frozen topology can be determined from the initial slope of $f(q, t)$. This analysis is shown in Figs. 1(d) and 1(f) for the $S q$ and $L q$ ranges, in which the faster decay is clearly detected. Dashed lines are exponential functions $f(q, t)=\exp \left(-t / \tau_{F}\right)$ fitting the initial decay of $f(q, t)$. The characteristic times obtained in this way are plotted for $T=27^{\circ} \mathrm{C}$ in Fig. $1(\mathrm{~g})$ as open symbols. As clearly visible, $\tau_{F}$ determined in the $L q$ and $S q$ grow proportionally to $q^{-2}$ and lay on the same line $\tau_{F}=A_{F} q^{-2}$ with $A_{F} \approx 10^{10} \mathrm{~s} / \mathrm{m}^{2}$ (dotted line). The same diffusive behavior is found at $T=35^{\circ} \mathrm{C}$, with similar $A_{F}$ coefficient (dotted line in Fig. 2).

Two distinct kinetic regimes can thus be identified. In the small $q$ region $\left(q<q_{c}\right)$ the DNA NS dynamics is formed by two coexisting mechanisms with $q^{-2}$ relaxation. When $q>q_{c}$, in a range where lengths are still much larger than the single NS, we find a distinct regime in which the relaxation is enabled by topological rearrangements of the DNA NS network. The challenge in interpreting this "topological regime" is to understand how local unbinding and binding of DNA NS affect in the same way-same kinetics - the onset and dissipation of concentration fluctuation that differ in size more than an order of magnitude.

Following each process of disruption and formation of bonds, the connectivity of the network locally changes. The well-studied case of the random-network structure of vitreous silica clarified that such tetrahedrally coordinated systems can form a large variety of topological patterns with quite different loop length distributions [20,21], yielding a significantly inhomogenous elastic response [22]. In the case of DNA NS, a system having a larger bond flexibility than silica [23] and with a ( $T$ dependent) fraction of unbound terminals, the accessible topologies cannot but be even more diverse. Thus, as NS tips unbind and rebind to available nearby open ends (a process requiring a minimal particle displacement), local connectivity is modified. Bulk modulus of gels crucially depends on the local connectivity and loop length [4], an old notion that recently found experimental confirmation [24]. Therefore, the transient DNA NS hydrogel is effectively a medium with a locally fluctuating elastic modulus, as sketched in Fig. 3(a), in which the local tightening or loosening of the network brings about a contraction or expansion that propagates in the system through a viscoelastic relaxation. Differently from particle diffusion phenomena, in which mass conservation implicitly implies $\tau \propto q^{-2}$, the process of elastic contraction or expansion couples to the concentration fluctuations via the change of the local volume. It is precisely the absence of a particle conservation law on a local scale that opens up the possibility of observing $q$-independent relaxation phenomena.

Such interplay between elasticity fluctuations and viscoelastic relaxation can be better understood by considering a simple 1D chain of beads and springs in which the spring constants $k_{n}$, mimicking the local elastic modulus, are allowed to fluctuate. Each choice of the set of elastic constants $\left\{k_{n}\right\}$ corresponds then to a given degree of frozen-in spatial disorder, which can be relaxed by reconfiguring the value of the elastic springs. For simplicity, we assume that each elastic constant $k_{n}$ fluctuates only between two equiprobable values $K_{1}$ and $K_{2}$ with an 


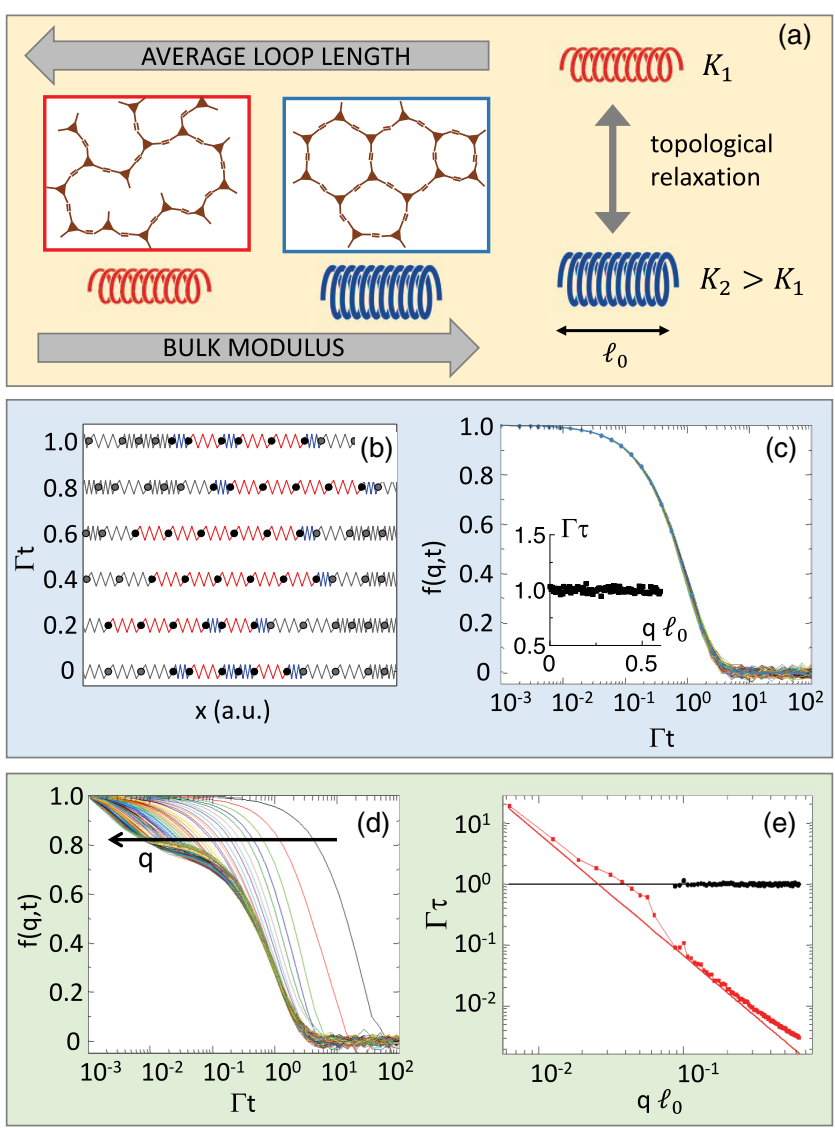

FIG. 3. Elasticity fluctuation model. (a) Networks of DNA NS can adopt various topological patterns, characterized by different loop lengths, resulting in different local bulk moduli, as described pictorially in the sketches. Topological relaxation occurring upon binding and unbinding of NS effectively provide fluctuations in the local elasticity, described in the 1D beads and spring model as a switching between elastic constants $K_{1}$ and $K_{2}$. (b) and (c) Elasticity fluctuation model for instantaneous relaxation of the bead positions. (b) Sequence of equilibrium configuration of 7 beads at the center of a string of 500 beads and springs. Time expressed in units of $\Gamma^{-1}$, the average spring refresh time. (c) Bead density correlation function $f(q, T)$ (different colors indicate different $q$ ) and their characteristic times vs $q$ (expressed in units of $1 / \ell_{0}$, the mean interbead distance). (d) $f(q, T)$ as in pane (c) calculated in the presence of thermal noise. (e) $q$ dependence of the two characteristic times $\tau_{1}$ (black dots) and $\tau_{2}$ (red dots). Black line: $\Gamma \tau=1$. Red line: viscoelastic relaxation time $\tau_{E}$ multiplied by $\Gamma$.

average refresh rate $\Gamma$. The beads density-density correlation function can be computed by their coordinates $\left\{r_{n}\right\}$ (see the Supplemental Material [18]).

We describe the model predictions in steps. We start by assuming that after each change in $\left\{k_{n}\right\}$ the system instantly evolves to the new equilibrium $\left\{r_{n}\right\}$. An example of the motions of a set of selected beads within the 1D array following spring refresh and fast equilibration is given in the drawing in Fig. 3(b). The corresponding $F(q, t)$, shown in Fig. 3(c), can be well described by exponential decays. It is evident from the collapse of the correlations and from the $q$ dependence of their decay times (in the inset), that this mechanism provides an identical relaxation at all $q$. The characteristic time is in all cases equal to $\Gamma^{-1}$, which marks the time requested to completely regenerate an uncorrelated sequence of spring constants. Indeed, this simple exercise clearly shows that spatially uncorrelated elasticity fluctuation uncoupled to particle transport gives rise to a length-scale independent decay of the concentration fluctuations over all lengths.

We then considered the same model in the presence of a thermal bath, inducing viscous damping and a random force acting on each bead. The corresponding equation of motion in the overdamped limit, appropriate to the dynamics of an aqueous gel, is now

$-k_{n}\left(r_{n}-r_{n-1}-l_{0}\right)+k_{n+1}\left(r_{n+1}-r_{n}-l_{0}\right)-\gamma \dot{r}_{n}+f_{n}=0$,

where $\gamma$ is the viscous damping on the beads and $f_{n}$ the delta-correlated random force acting on bead $n$, whose amplitude is fixed by the dissipation-fluctuation theorem. Solutions of Eq. (2) when all $k_{n}$ are equal $\left(k_{n}=K\right)$ and fixed to their initial value shows that the density fluctuations relax to a nonergodicity plateau with a viscoelastic time $\tau_{E}=(\gamma / K)\left(q l_{0}\right)^{-2}$, e.g., a time which grows with $q^{-2}$ as in the case of the phonon dispersion relation in colloidal crystals $[25,26]$.

We finally move to the relevant case in which the $\left\{k_{n}\right\}$ fluctuate between $K_{1}$ and $K_{2}$. Now at each integration time step $d t$ the value of all $\left\{k_{n}\right\}$ is reassigned with probability $\Gamma d t$. The decay of the numerically evaluated $F(q, t)$ [Fig. 3(d)] features two distinct relaxation processes that merge as $q$ decreases. At larger $q$, the two modes have a clearly distinct dependence on $q$ [Fig. 3(e)]. The slower $\left(\tau_{1}\right)$ is $q$ independent, and $\tau_{1} \approx \Gamma^{-1}$. The faster $\left(\tau_{2}\right)$ is instead $q$ dependent, with value and slope approximately matching those of $\tau_{E}$, where $K=\left(K_{1}+K_{2}\right) / 2$ is now the average elastic constant. Figure 3(e) shows that as $\tau_{E}$ approaches $\Gamma^{-1}$, the slower relaxation time exhibits a crossover from a $q^{0}$ regime at larger $q$ to a $q^{-2}$ regime at the limit of small $q$. This behavior confirms the notion introduced above: when the reconfiguration of the network takes place on time scales faster than the damped elastic propagation, the system relaxation is length-scale independent. When instead the viscoelastic propagation of the elastic changes is slower than the network reconfiguration, the dominant time is the viscoelastic behavior with the expected diffusive $q^{-2}$ dependent dynamics. This simple model offers a microscopic understanding of the de Gennes' "two fluid model" $[1,10,27]$, which was proposed to account for the complex kinetics of a polymeric network by introducing an effective Maxwell-type frequency-dependent viscoelastic response of the network $M(\omega)$, which, however, had no reference to molecular processes. Indeed, it is possible to show that our model yields a viscoelastic response analogous to $M(\omega)$.

As a further check of the appropriateness of the fluctuating elasticity model for our system, it is also possible to compute 
a rough estimate of $\tau_{E}$ for the NP network (see the Supplemental Material [18]). We obtain that the expected $\tau_{E}$ should be $\tau_{E}>0.02 q^{-2}[s]$. This estimate is in the same order of magnitude of the experimental observation and it is reasonably compatible with both observed $q^{-2}$ modes. Such ambiguity cannot be further disentangled by the beads and spring model, which explains the $q^{0}$ mode and its crossover to a diffusion process, but cannot reproduce the entire experimental findings. Indeed, in the limit of small $q$ the model predicts only one detectable relaxation process. This is expected since we have included neither thermal diffusion nor the small but not negligible free particle diffusion, which are crucial for establishing the correct long wavelengths limits. Memory function or appropriate conservation laws (in the manner of Navier and Stokes) could provide more accurate (but perhaps less transparent) descriptions.

Conclusions. - We have studied the anomalous lengthscale-independent relaxation of a DNA-based transient network. The strong $T$ dependence of the lifetime of the interparticle bonds makes it possible to experimentally access the crossover between the diffusive $\left(q^{-2}\right)$ and $q$ independent $\left(q^{0}\right)$ modes. By leveraging on the detailed knowledge of the system, we pinpoint the origin of the $q^{0}$ mode in the local topological reconfiguration of the network and the resulting fluctuation of the network's local bulk modulus. The notion of independent elastically relaxing local units introduces a mechanism of concentration fluctuation which does not require overall mass transport, a crucial requirement to preempt the application of the particle conservation law and to give rise to a $q^{0}$ mode. This notion also naturally introduces a crossover between the time scale of local elasticity fluctuations and the time scale of viscoelastic propagation, which becomes the dominant decorrelation mechanism at large length scales. This behavior is captured and explained by a simple 1D model based of beads and springs with fluctuating elastic constants. The model, however, does not reproduce the presence of the two distinct diffusive modes which we observe at low $q$. Further theoretical modeling is thus required to explain the origin of an additional mode and its possible interactions with the fluctuating elastic mode.

Our findings reveal the intimate connection between the reversibility of the bonds and the $q^{0}$ mode, which thus emerges as a universal property of the dynamics of transient networks. Even more generally, the $q^{0}$ behavior could be a feature typical of all systems having a slow dynamics caused by independently relaxing distinct regions, with its expected range at wavelengths larger than the characteristic size of the relaxing units.

We thank T. Franosh, F. Giavazzi, L. Rovigatti, and M. Rubinstein for discussions. We acknowledge support from the Italian Ministry of Education and Research, projects PRIN (2010LKE4CC "Building Materials with DNA bricks"). *tommaso.bellini@unimi.it

[1] P. G. De Gennes, Macromolecules 9, 587 (1976).

[2] M. D. Hager, P. Greil, C. Leyens, S. van der Zwaag, and U. S. Schubert, Adv. Mater. 22, 5424 (2010).

[3] D. Montarnal, M. Capelot, and F. Tournilhac, Science 334, 965 (2011).

[4] M. Rubinstein and R. Colby, Polymer Physics (Oxford University Press, New York, 2003).

[5] A. Sonn-Segev, A. Bernheim-Groswasser, H. Diamant, and Y. Roichman, Phys. Rev. Lett. 112, 088301 (2014).

[6] F. Herbst, K. Schröter, I. Gunkel, S. Gröger, T. ThurnAlbrecht, J. Balbach, and W. H. Binder, Macromolecules 43, 10006 (2010).

[7] U. Peter, D. Roux, and A. K. Sood, Phys. Rev. Lett. 86, 3340 (2001).

[8] N. Nemoto, A. Koike, and K. Osaki, Macromolecules 29, 1445 (1996).

[9] A. Koike, N. Nemoto, T. Inoue, and K. Osaki, Macromolecules 28, 2339 (1995).

[10] M. Adam and M. Delsanti, Macromolecules 18, 1760 (1985).

[11] S. Dai, K. C. Tam, and R. D. Jenkins, Macromol. Chem. Phys. 203, 2312 (2002).

[12] N. Nemoto, M. Kuwahara, M. Yao, and K. Osaki, Langmuir 11, 30 (1995).

[13] E. Michel, M. Filali, R. Aznar, G. Porte, and J. Appell, Langmuir 16, 8702 (2000).

[14] R. Johannsson, C. Chassenieux, D. Durand, T. Nicolai, P. Vanhoorne, and R. Jerome, Macromolecules 28, 8504 (1995).

[15] J. Li, T. Ngai, and C. Wu, Polym. J. 42, 609 (2010).

[16] S. Biffi, R. Cerbino, F. Bomboi, E. M. Paraboschi, R. Asselta, F. Sciortino, and T. Bellini, Proc. Natl. Acad. Sci. U.S.A. 110, 15633 (2013).

[17] E. Bianchi, J. Largo, P. Tartaglia, E. Zaccarelli, and F. Sciortino, Phys. Rev. Lett. 97, 168301 (2006).

[18] See Supplemental Material at http://link.aps.org/ supplemental/10.1103/PhysRevLett.119.078002 for details on DNA sequences, experimental setup, correlation signal analysis and an appendix to the theoretical model proposed in the main text.

[19] S. Biffi, R. Cerbino, G. Nava, F. Bomboi, F. Sciortino, and T. Bellini, Soft Matter 11, 3132 (2015).

[20] L. Lichtenstein, C. Büchner, B. Yang, S. Shaikhutdinov, M. Heyde, M. Sierka, R. Włodarczyk, J. Sauer, and H.-J. Freund, Angew. Chem., Int. Ed. 51, 404 (2012).

[21] G. Pacchioni, L. Skuja, and D. L. Griscom eds., Defects in SiO2 and Related Dielectrics: Science and Technology (Springer Netherlands, Dordrecht, 2000).

[22] F. Leonforte, A. Tanguy, J. P. Wittmer, and J. L. Barrat Phys. Rev. Lett. 97, 055501 (2006).

[23] I. Saika-Voivod, F. Smallenburg, and F. Sciortino, J. Chem. Phys. 139, 234901 (2013).

[24] M. Zhong, R. Wang, K. Kawamoto, B. D. Olsen, and J. A. Johnson, Science 353, 1264 (2016).

[25] A. J. Hurd, N. A. Clark, R. C. Mockler, and W. J. O'Sullivan, Phys. Rev. A 26, 2869 (1982).

[26] J. Derksen and W. van de Water, Phys. Rev. A 45, 5660 (1992).

[27] F. Brochard, J. Phys. (Paris), Lett. 44, 39 (1983). 